\title{
Chapter 141 \\ Report on Global Environment \\ Competitiveness of the United States
}

The United States of America is a federal constitutional republic comprising 50 states and a federal district. The country is situated mostly in central North America, where its 48 contiguous states and Washington, D.C., lie between the Pacific and Atlantic Oceans, bordered by Canada to the north and Mexico to the south. It covers $9,147.4$ thousand of square kilometers and has a population of 311.59 million. Its GDP reaches $\$ 14,991.30$ billion in 2011 . Through the index system and evaluation model, the environment competitiveness index of The United States ranks at 26 in 133 countries.

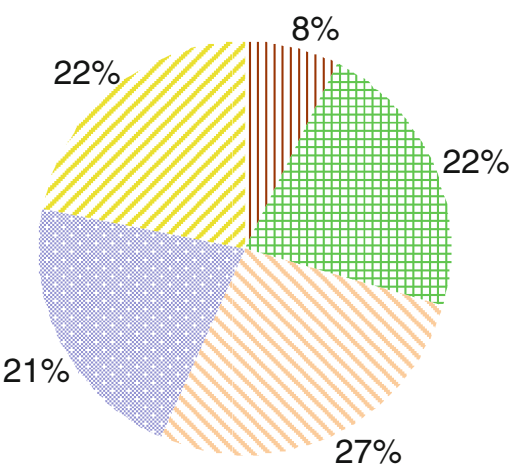

III Resource Environment Competitiveness

\#\# Ecological Environment Competitiveness Environment Carrying Competitiveness

Fig. 141.1 Contribution of

Environment Management Competitiveness Environment Harmony sub-index to GEC Competitiveness 


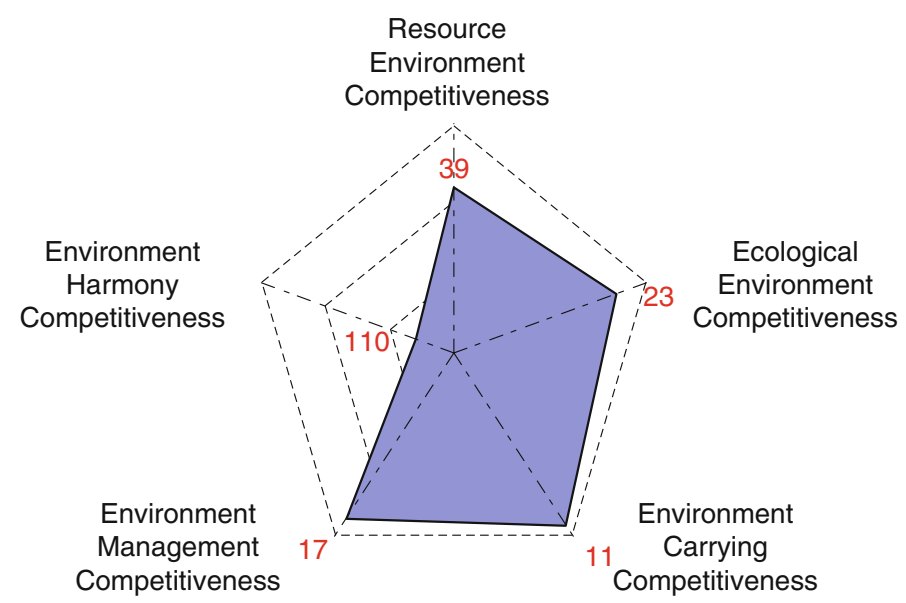

Fig. 141.2 Rank of sub-index of GEC

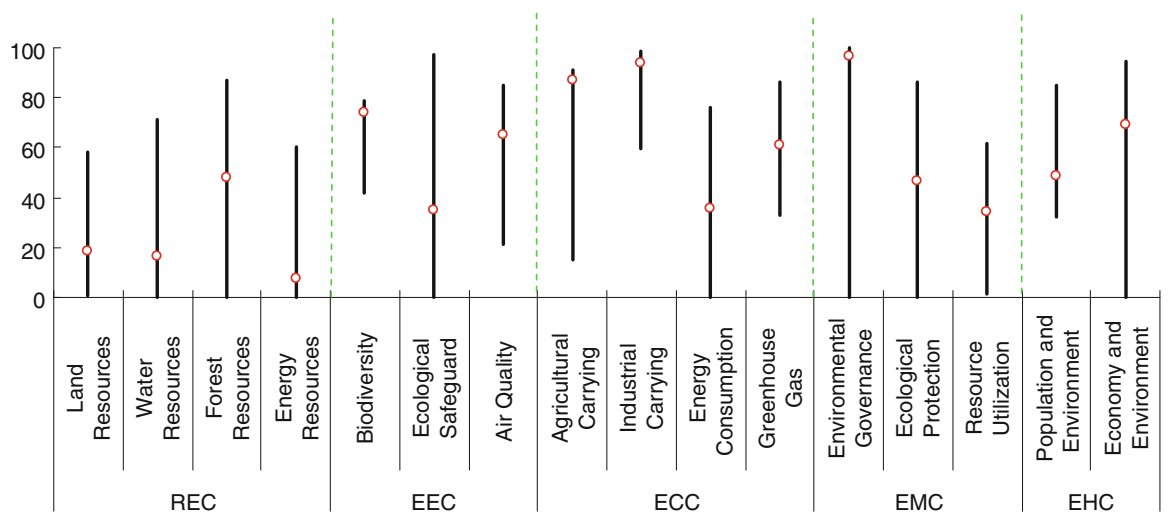

Fig. 141.3 Score and rank of the pillars of GEC

Table 141.1 Score and rank of all indicators of GEC

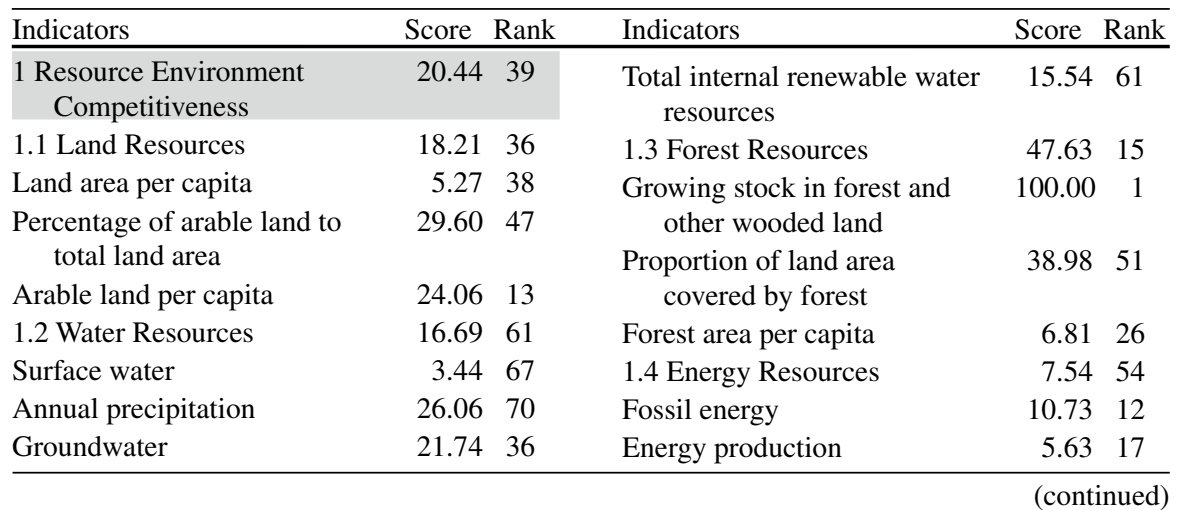


Table 141.1 (continued)

\begin{tabular}{|c|c|c|c|c|c|}
\hline Indicators & Score & Rank & Indicators & Score & Rank \\
\hline $\begin{array}{l}\text { Proportion of combustible } \\
\text { renewable and waste to }\end{array}$ & 4.37 & 89 & $\begin{array}{l}\text { Ratio of clean energy } \\
\text { consumption }\end{array}$ & 20.22 & 32 \\
\hline total energy consumption & & & Elasticity of energy & 13.48 & 96 \\
\hline $\begin{array}{l}\text { Net energy imports of the } \\
\text { energy consumption }\end{array}$ & 9.83 & 61 & \multirow{2}{*}{$\begin{array}{l}\text { Elasticity of electric power } \\
\text { consumption }\end{array}$} & \multirow{2}{*}{\multicolumn{2}{|c|}{8.46111}} \\
\hline 2 Ecological Environment & 58.67 & 23 & & & \\
\hline $\begin{array}{l}\text { Competitiveness } \\
\text { 2.1 Biodiversity }\end{array}$ & 73.84 & 3 & $\begin{array}{l}\text { 3.4 Greenhouse Gas } \\
\text { Growth rate of } \mathrm{CO}_{2} \text { emissions }\end{array}$ & $\begin{array}{l}61.16 \\
52.61\end{array}$ & $\begin{array}{l}71 \\
62\end{array}$ \\
\hline Threatened fish species & 13.68 & 132 & Growth rate of Methane & 66.54 & 18 \\
\hline Threatened mammal species & 79.89 & 115 & emissions & & \\
\hline Threatened plant species & 87.22 & 122 & $\mathrm{CO}_{2}$ emissions per unit of land & 99.35 & 105 \\
\hline $\begin{array}{l}\text { GEF benefits index for } \\
\text { biodiversity }\end{array}$ & 94.20 & 2 & $\begin{array}{l}\text { area } \\
\mathrm{CO}_{2} \text { emissions per unit of }\end{array}$ & 34.71 & 88 \\
\hline 2.2 Ecological Safeguard & 35.19 & 32 & energy consumption & & \\
\hline Terrestrial protected areas & 33.42 & 58 & $\begin{array}{l}4 \text { Environment Management } \\
\text { Competitiveness }\end{array}$ & 57.79 & 17 \\
\hline $\begin{array}{l}\text { Marine protected areas } \\
\text { 2.3 Air Quality }\end{array}$ & $\begin{array}{l}37.85 \\
64.90\end{array}$ & $\begin{array}{r}8 \\
55\end{array}$ & $\begin{array}{l}\text { Competitiveness } \\
\text { 4.1 Environmental Governance }\end{array}$ & 96.30 & 35 \\
\hline Inhalable particles (PM10) & 86.86 & 30 & Agricultural chemicals & 95.24 & 20 \\
\hline Particulate matter (PM2.5) & 86.68 & 57 & regulation & & \\
\hline Index of indoor air pollution & 100.00 & 1 & Percentage of the rural & 94.00 & 49 \\
\hline Nitrogen oxides emission & 1.12 & 131 & $\begin{array}{l}\text { population with access to } \\
\text { an improved water source }\end{array}$ & & \\
\hline Sulfur dioxide emission & 0.13 & 130 & Percentage of the urban & 100.00 & 1 \\
\hline $\begin{array}{l}3 \text { Environment Carrying } \\
\text { Competitiveness }\end{array}$ & 73.42 & 11 & $\begin{array}{l}\text { population with access to } \\
\text { an improved water source }\end{array}$ & & \\
\hline 3.1 Agricultural Carrying & 86.67 & 3 & 4.2 Ecological Protection & 46.74 & 19 \\
\hline $\begin{array}{l}\text { Cereal yield per unit of arable } \\
\text { land }\end{array}$ & 74.68 & 7 & $\begin{array}{l}\text { Area of plantation and } \\
\text { afforestation }\end{array}$ & 32.87 & 2 \\
\hline $\begin{array}{l}\text { Fertilizer consumption per unit } \\
\text { of arable land }\end{array}$ & 91.16 & 84 & Biome protect & 49.40 & 73 \\
\hline Annual freshwater withdrawals & 98.17 & 72 & $\begin{array}{l}\text { Overfishing of fishing } \\
\text { resources }\end{array}$ & 62.59 & 31 \\
\hline $\begin{array}{l}\text { for agriculture per unit of } \\
\text { arable land }\end{array}$ & & & 4.3 Resource Utilization & 34.02 & 76 \\
\hline 3.2 Industrial Carrying & 93.68 & 36 & $\begin{array}{l}\text { Utilization rate of water } \\
\text { resources }\end{array}$ & 0.63 & 51 \\
\hline $\begin{array}{l}\text { Net exports of goods as a } \\
\text { percentage of GDP }\end{array}$ & 93.80 & 7 & $\begin{array}{l}\text { Percentage of total internal } \\
\text { renewable water resources }\end{array}$ & 65.60 & 64 \\
\hline Electric power consumption & 89.86 & 86 & to total water resources & & \\
\hline industry & & & $\begin{array}{l}\text { Percentage of agricultural land } \\
\text { to total land area }\end{array}$ & 52.13 & 63 \\
\hline $\begin{array}{l}\mathrm{SO}_{2} \text { emissions per unit of value } \\
\text { added of industry }\end{array}$ & 99.87 & 48 & Percentage of fossil fuel & 17.72 & 78 \\
\hline Annual freshwater withdrawals & 91.20 & 113 & $\begin{array}{l}\text { energy consumption to total } \\
\text { energy consumption }\end{array}$ & & \\
\hline added of industry & & & $\begin{array}{l}5 \text { Environment Harmony } \\
\text { Competitiveness }\end{array}$ & 58.82 & 110 \\
\hline $\begin{array}{l}\text { 3.3 Energy Consumption } \\
\text { Energy consumption per unit } \\
\text { of land area }\end{array}$ & $\begin{array}{l}35.41 \\
99.49\end{array}$ & $\begin{array}{l}42 \\
98\end{array}$ & $\begin{array}{l}\text { 5.1 Population and } \\
\text { Environment }\end{array}$ & 48.48 & 128 \\
\hline
\end{tabular}


Table 141.1 (continued)

\begin{tabular}{|c|c|c|c|}
\hline Indicators & Score Rank & Indicators & Score Rank \\
\hline \multirow{2}{*}{$\begin{array}{l}\text { Percentage of population with } \\
\text { access to Improved } \\
\text { sanitation facilities }\end{array}$} & \multirow[t]{2}{*}{100.00} & 5.2 Economy and En & $69.16 \quad 34$ \\
\hline & & $\begin{array}{l}\text { Land resource utilization } \\
\text { efficiency }\end{array}$ & $0.48 \quad 29$ \\
\hline \multirow{3}{*}{$\begin{array}{l}\text { Motor vehicles per } 1,000 \text { people } \\
\text { Renewable internal freshwater } \\
\text { resources per capita }\end{array}$} & 0.00133 & \multirow{2}{*}{$\begin{array}{l}\text { Sulfur dioxide emissions per } \\
\text { unit of GDP }\end{array}$} & \multirow[t]{2}{*}{$97.02 \quad 38$} \\
\hline & $10.94 \quad 35$ & & \\
\hline & & \multirow{2}{*}{$\begin{array}{l}\text { Carbon dioxide emissions per } \\
\text { unit of GDP }\end{array}$} & \multirow[t]{2}{*}{$87.69 \quad 68$} \\
\hline $\mathrm{SO}_{2}$ emissions per capita & 81.24112 & & \\
\hline $\mathrm{CO}_{2}$ emissions per capita & 53.14128 & \multirow{2}{*}{$\begin{array}{l}\text { Energy consumption per unit } \\
\text { of GDP }\end{array}$} & \multirow[t]{2}{*}{91.46} \\
\hline Energy consumption per capita & 44.52120 & & \\
\hline
\end{tabular}

Table 141.2 Rank distribution of the individual indicators of GEC

\begin{tabular}{|c|c|c|c|c|c|c|}
\hline Sub-index & $\begin{array}{l}\text { Number of } \\
\text { the individual } \\
\text { indicators }\end{array}$ & $\begin{array}{l}\text { Rank } \\
1-10\end{array}$ & $\begin{array}{l}\text { Rank } \\
11-30\end{array}$ & $\begin{array}{l}\text { Rank } \\
31-60\end{array}$ & $\begin{array}{l}\text { Rank } \\
61-100\end{array}$ & $\begin{array}{l}\text { Rank } \\
101-133\end{array}$ \\
\hline $\begin{array}{l}\text { Resource Environment } \\
\text { Competitiveness }\end{array}$ & 14 & 1 & 3 & 6 & 4 & 0 \\
\hline $\begin{array}{l}\text { Ecological Environment } \\
\text { Competitiveness }\end{array}$ & 11 & 2 & 1 & 4 & 0 & 4 \\
\hline $\begin{array}{c}\text { Environment Carrying } \\
\text { Competitiveness }\end{array}$ & 15 & 3 & 1 & 4 & 6 & 1 \\
\hline $\begin{array}{l}\text { Environment Management } \\
\text { Competitiveness }\end{array}$ & 10 & 1 & 2 & 3 & 4 & 0 \\
\hline $\begin{array}{c}\text { Environment Harmony } \\
\text { Competitiveness }\end{array}$ & 10 & 1 & 1 & 3 & 1 & 4 \\
\hline Total & 60 & 8 & 8 & 20 & 15 & 9 \\
\hline
\end{tabular}

Open Access This chapter is distributed under the terms of the Creative Commons Attribution Noncommercial License, which permits any noncommercial use, distribution, and reproduction in any medium, provided the original author(s) and source are credited. 